\title{
DISTRIBUIÇÃO E ABUNDÂNCIA DE ISÓPODOS TERRESTRES (CRUSTACEA: ISOPODA: ONISCIDEA) NAS MARISMAS DO ESTUÁRIO DA LAGOA DOS PATOS, RIO GRANDE DO SUL, BRASIL
}

\author{
ELIS REGINA LOPES-LEITZKEE ${ }^{1}$, CÉSAR SERRA BONIFÁCIO COSTA², CRISTINA MARIA LOYOLA ZARDO ${ }^{3}$, FERNANDO D'INCAO ${ }^{4}$ \\ ${ }^{1}$ Universidade Federal do Rio Grande, Instituto de Ciências Biológicas - ICB. Laboratório de Zoologia. \\ CEP: 96201900 - Rio Grande, RS - Brasil - Caixa-postal: 474. E-mail: elisleitzke@gmail.com \\ ${ }^{2}$ Universidade Federal do Rio Grande, Instituto de Oceanografia, Laboratório de Biotecnologia de Halófitas. Carreiros \\ Cep: 96203900 - Rio Grande, RS - Brasil - Caixa-postal: 474 \\ ${ }^{3}$ Universidade Federal do Rio Grande, Instituto de Ciências Biológicas. Avenida Italia, km 8 - Pavilhão 6 - Sala 22G \\ Carreiros. Cep: 96201090 - Rio Grande, RS - Brasil - Caixa-postal: 474 \\ ${ }^{4}$ Universidade Federal do Rio Grande, Departamento de Oceanografia, Laboratório de Crustáceos Decápodos. \\ Instituto de Oceanografia - FURG, Av. Itália Km 8 Carreiros. Cep: 96201-900 - Rio Grande, RS - Brasil - Caixa-postal: 474
}

\begin{abstract}
RESUMO
Marismas são habitats abioticamente estressantes, mas altamente produtivos. A maior parte desta produção é consumida por detritívoros, como os Oniscidea. O trabalho objetivou conhecer a abundância e a distribuição de isópodos terrestres nas marismas do Estuário da Lagoa dos Patos, Brasil. Três marismas foram amostradas na primavera/2007, verão, outono e inverno/2008. Em cada marisma, um transecto de $10 \mathrm{~m}$ foi fixado, onde 10 quadrados de $20 \times 20 \mathrm{~cm}$ foram colocados e todo material removido. A variação de abundância das espécies foi comparada entre as marismas através de Manova. Duas espécies foram identificadas em cada marisma: Balloniscus sellowii e Atlantoscia floridana na Barra e Pólvora e B. sellowii e B. glaber na Torotama. A densidade média de isópodos encontrada na Barra foi de $6,9 \mathrm{ind} / \mathrm{m}^{2}$, na Pólvora de $5,0 \mathrm{ind} / \mathrm{m}^{2}$ e na Torotama de $6,1 \mathrm{ind} / \mathrm{m}^{2}$. A abundância de isópodos terrestres foi estatisticamente diferente entre as estações do ano $(p=0,00)$, mas não entre as marismas $(p=$ 0,86 ). Na Torotama/Inverno, a abundância de isópodos é significativamente maior do que nas demais estações e locais, exceto na Barra/Primavera $(p=0,14)$; Barra/Inverno $(p=0,20)$ e Pólvora/Primavera $(p=0,38)$. A salinidade é inversamente proporcional ao número de indivíduos coletados na Barra e na Pólvora. Na Torotama, o número de isópodos cresce com o aumento do nível da água e do detrito depositado sobre o terreno. A distribuição e abundância de isópodos terrestres são fortemente influenciadas por fatores ambientais.
\end{abstract}

PALAVRAS-CHAVE: marismas, ecologia, Oniscidea, estuário, abundância

\section{ABSTRACT}

Saltmarshes are abiotically stressful habitats, but highly productive. Most of this production is consumed by detritivorous such as Oniscidea. The study focused on the abundance and distribution of terrestrial isopods in the saltmarshes of the Estuary of the Patos Lagoon, Brazil. Three saltmarshes were sampled in spring/2007, summer, autumn and winter/2008. In each saltmarsh, a transect of $10 \mathrm{~m}$ was set, where 10 squares of the $20 \times 20 \mathrm{~cm}$ were placed and all material removed. The variation of species abundance was compared between the saltmarshes through Manova. Two species were identified in each saltmarsh: Balloniscus sellowii and Atlantoscia floridana in West Breakwater of Rio Grande Channel and in Polvora Island and B. sellowii and $B$. glaber in Torotama Island. The mean density of isopods was $6.9 \mathrm{ind} / \mathrm{m}^{2}$ (West Breakwater), $5.0 \mathrm{ind} / \mathrm{m}^{2}$ (Polvora) and 6.1 ind $/ \mathrm{m}^{2}$ (Torotama). The abundance of terrestrial isopods was statistically different between the seasons $(p=0.00)$ but not between the saltmarshs $(p=0.86)$. In Torotama/winter, abundance of isopods is significantly higher than in other seasons and sites, except in the West Breakwater/spring $(p=0.14)$, West Breakwater/winter $(p=0.20)$ and Polvora/Spring $(p=0.38)$. The salinity is inversely proportional to the number of individuals in West Breakwater and Polvora. In Torotama, the number of isopods increases with the increase in the level of water and detritus deposited on the ground. The distribution and abundance of terrestrial isopods are strongly influenced by environmental factors.

KEY WORDS: saltmarsh, ecology, Oniscidea, estuary, abundance

\section{INTRODUÇÃO}

A combinação de submersão e exposição periódicas, variabilidade osmótica do solo e água intersticial e flutuações de temperatura fazem das marismas ambientes mais ríspidos do que os ambientes marinho e terrestre (CHAPMAN 1992; VERNBERG 1993). Entretanto, as marismas apresentam uma elevada produtividade primária, com as plantas e animais que aí se encontram adaptados aos extremos ambientais (GAONA et al. 1996; SEELIGER et al. 1997; COSTA 1998a).

As maiores extensões de marismas da costa brasileira são observadas nos estados de Santa Catarina (PANITZ 1992) e do Rio Grande do Sul (COSTA et al. 1997), onde neste último, 95\% da área de marisma encontram-se no extremo sul, junto aos municípios de Rio Grande e São José do Norte (COIMBRA \& COSTA 2006). Apesar dos diversos estudos sobre a produtividade primária das extensas marismas do Estuário da Lagoa dos Patos (GAONA et al. 1996; COSTA 1998a; 1998b), pouco é ainda conhecido sobre suas inter-relações com o corpo lacunar e águas costeiras adjacentes (COSTA \& DAVY 1992; SILVA et al. 1993; HICKENBICK et al. 2004).

As marismas são dominadas por plantas de origem terrestre, mas contém animais de origem marinha e terrestre, o que as caracterizam como ligação evolutiva e ecológica entre ambos os ecossistemas (ZIMMER et al. 2002). Comunidades em zonas entre o nível médio das marés e a borda superior das marismas atingida apenas pelas marés sizígias, 
são dominadas por espécies de detritívoros de origem terrestre, incluindo isópodos, que podem alimentar-se de detritos derivados tanto das angiospermas das marismas quanto de plantas terrestres das outras regiões (ZIMMER 2003).

Diferentes espécies de isópodos terrestres têm sido citadas como componentes importantes na comunidade de artrópodos fragmentadores da matéria orgânica particulada em marismas de todo o mundo (TEAL 1962; ADAM 1993; LEVIN \& TALLEY 2000; KREEGER \& NEWELL 2000; ZIMMER 2003; ZIMMER et al. 2002; DIAS et al. 2005).

Mundialmente, a fauna de isópodos terrestres associada as marismas ainda está pouco estudada, principalmente, em relação a ocorrência e comportamento em um ambiente com condições abióticas tão variáveis. TALLEY et al. (2001), verificaram a utilização do habitat e a alteração causada pelo isópodo escavador, Sphaeroma quoyanum, em uma marisma da Califórnia. ZIMMER et al. (2002), testaram os padrões de consumo e decomposição de detritos vegetais pelos isópodos terrestres em uma marisma alta e uma floresta costeira, na llha Sapelo, Geórgia. Mais recentemente, a abundância e a história de vida de quatro espécies de isópodos terrestres (Tylos ponticus, Porcellio lamellatus, Halophiloscia couchii e Armadillidium album) foram analisadas em uma marisma do sistema de lagoas do Rio Formosa, Portugal, por DIAS et al. (2005).

Quanto as marismas do Estuário da Lagoa dos Patos, no sul do Brasil, GAONA et al. (1996), verificaram as flutuações mensais nas taxas de perdas de biomassa morta, na comunidade de Juncus effusus (atualmente $\mathrm{J}$. kraussii) e relacionaram estas com a presença dos isópodos terrestres Balloniscus sellowii e Balloniscus glaber, os quais atuariam no processo de consumo e fragmentação de detritos. COSTA et al. (1997) identificaram os Oniscidea, entre eles representantes do gênero Balloniscus, como invertebrados que se beneficiam destas marismas.

Desta forma, o trabalho visou conhecer a fauna e a abundância de isópodos terrestres nas marismas do Estuário da Lagoa dos Patos, observando a variação sazonal das espécies e a sua relação com a cobertura vegetal, a abundância de detrito e parâmetros ambientais.

\section{MATERIAL E MÉTODOS}

O estudo foi realizado em três marismas distintas quanto aos valores médios de salinidade da água de alagamento, no Estuário da Lagoa dos Patos, Rio Grande, Rio Grande do Sul, Brasil: Molhe Oeste da Barra de Rio Grande (32 $\left.09^{\prime} 07.75^{\prime \prime} 55^{\circ} 06^{\prime} 11.87^{\prime \prime} \mathrm{W}\right)$, Ilha da Pólvora (3201'09.83"S 52 $\left.06^{\prime} 16.85^{\prime \prime W}\right)$ e llha da Torotama (31 ${ }^{\circ} 54^{\prime} 41.37^{\prime \prime S} 52^{\circ} 10^{\prime} 43.85^{\prime \prime W}$ ) (Fig. 1). Os pontos amostrais estavam localizados, respectivamente, numa distância de 160 m, $110 \mathrm{~m} \mathrm{e}$ $500 \mathrm{~m}$ da margem da Lagoa.

Em cada marisma, um transecto de $10 \mathrm{~m}$ de comprimento foi fixado por estacas, no centro de três diferentes habitats com distinta cobertura vegetal (COSTA et al. 2003). Foram amostrados habitats de marisma baixa, dominada por Spartina alterniflora, marisma média, dominada por Spartina densiflora ou Scirpus maritimus e marisma alta, com sua cobertura vegetal dominada por Juncus kraussii ou mata de capororoca (dominância de Rapanea (Myrsine) parvifolia).

As coletas foram realizadas durante os meses de nov-dez/07 (primavera); jan-mar/08 (verão); abr/08 (outono) e jul-ago/08 (inverno). Duas coletas foram realizadas, nos diferentes habitats das marismas, em cada estação do ano.

Ao longo do transecto, 10 quadrados de $20 \times 20 \mathrm{~cm}$ foram colocados aleatoriamente e todo material vegetal vivo e morto e a epifauna foram removidos, constituindo uma coleta destrutiva. As medidas de $\mathrm{pH}$, salinidade e temperatura da água intersticial de cada ponto amostral foram tomadas. Para se registrar a condição de alagamento da marisma em cada coleta, foi medido o nível da água na margem de um marco colocado em cada local amostrado.

Em laboratório, os isópodos terrestres foram retirados manualmente, fixados em álcool 70ㅜ, identificados e depositados na coleção de Crustáceos (IO/FURG). Os dados de contagem de indivíduos de cada espécie de isópodo terrestre em cada tipo de cobertura vegetal foram utilizados como estimativa da densidade populacional por $\mathrm{m}^{2}$.

Após a retirada dos isópodos, a biomassa aérea vegetal de cada amostra foi separada em material vivo e morto (incluindo detrito depositado sobre o terreno), e 
ambos os componentes foram secos em estufa a $80^{\circ} \mathrm{C}$ por 48 horas, para a obtenção de peso constante $( \pm 0,01 \mathrm{~g})$. A biomassa aérea foi pesada em balança analítica, antes e depois da secagem.

Os dados receberam tratamentos estatísticos conforme ZAR (1999). A variação de abundância dos isópodos terrestres encontrados sazonalmente, foi comparada entre os hábitats, através de análise de variância multifatorial. Os relacionamentos entre parâmetros abióticos $(\mathrm{pH}$, temperatura, salinidade e nível da água), biomassa vegetal e abundância de isópodos, de cada marisma amostrada, foram analisados através de regressões múltiplas.

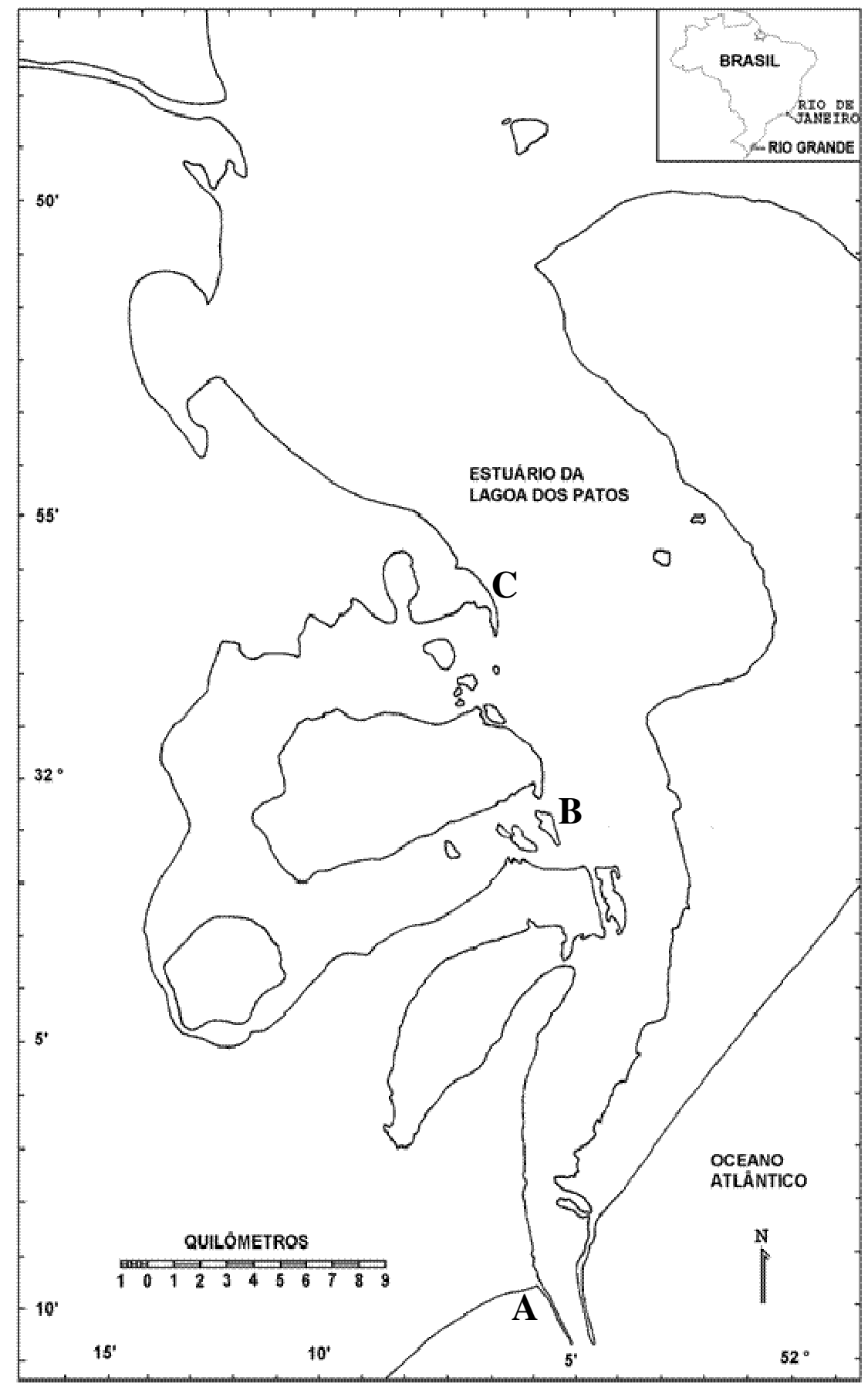

FIGURA 1 - Figura da área de estudo, indicando as três marismas localizadas no Estuário da Lagoa dos Patos, Rio Grande, Rio Grande do Sul, Brasil. A: Molhe Oeste da Barra de Rio Grande; B. Ilha da Pólvora e C. Ilha da Torotama. 


\section{RESULTADOS}

Um total de 470 quadrados foi amostrado ao longo das três marismas selecionadas para o estudo: 210 quadrados no Molhe Oeste da Barra de Rio Grande, 180 quadrados na llha da Pólvora e 80 quadrados na llha da Torotama, onde, devido a acessibilidade do local, apenas a marisma média ocasionalmente alagada e com sua cobertura vegetal dominada por $S$. densiflora foi amostrada. Apesar de encontrados nas quatro estações do ano, os isópodos não foram coletados em todos os hábitats dos três locais de estudo. Durante o período amostral, três espécies de Oniscidea, pertencentes a duas famílias, foram encontradas nas marismas do Estuário da Lagoa dos Patos, sendo elas: Família Balloniscidae Balloniscus sellowii (Brandt, 1833) e Balloniscus glaber Araujo e Zardo, 1995 e Família Philosciidae Atlantoscia floridana (van Name, 1940).

No Molhe Oeste, apenas no hábitat de marisma alta, raramente alagada e com sua cobertura vegetal dominada por $J$. kraussii foi registrada a presença de Oniscidea (194 indivíduos). Na llha da Pólvora, isópodos terrestres foram encontrados nos hábitats de marisma alta (121 indivíduos) e média, onde, nesta última, somente 07 indivíduos de uma mesma espécie foram coletados. Já na llha da Torotama, 195 exemplares foram registrados no habitat amostrado.
A Figura 2 mostra a variação do número de indivíduos das espécies coletadas, em cada marisma amostrada. Um total de 517 indivíduos foi coletado. Dentre as espécies, a mais abundante foi Balloniscus sellowii, com $87,62 \%$ dos indivíduos amostrados, seguido de Balloniscus glaber (10,83\%) e Atlantoscia floridana (1,55\%) (Tab. I). A densidade média de isópodos terrestres no Molhe Oeste foi de $6,9 \mathrm{ind} / \mathrm{m}^{2}$ $\left(6,72 \mathrm{ind} / \mathrm{m}^{2}\right.$ de $B$. sellowii e $0,22 \mathrm{ind} / \mathrm{m}^{2}$ de $A$. floridana), na Pólvora foi de $5,32 \mathrm{ind} / \mathrm{m}^{2}\left(4,95 \mathrm{ind} / \mathrm{m}^{2}\right.$ de $B$. sellowii na marisma alta, $0,12 \mathrm{ind} / \mathrm{m}^{2}$ de $B$. sellowii na marisma média e $0,07 \mathrm{ind} / \mathrm{m}^{2}$ de $A$. floridana na marisma alta) e na Torotama foi de 6,1 ind $/ \mathrm{m}^{2}\left(4,35 \mathrm{ind} / \mathrm{m}^{2}\right.$ de $B$. sellowii e $1,75 \mathrm{ind} / \mathrm{m}^{2}$ de $B$. glaber).

$\mathrm{Na}$ Figura 3, pode-se observar a variação da abundância média de Oniscidea nas três marismas amostradas ao longo das estações do ano. Nas marismas da Barra e da Pólvora, a abundância de isópodos terrestres diminui durante o verão e o outono, ao contrário do que se observa na Torotama, onde a abundância média dos indivíduos aumenta ao longo das estações, atingindo seu pico no inverno. A relação entre a abundância média de indivíduos coletados nas marismas amostradas e os valores médios calculados para os parâmetros ambientais e para a quantidade de detrito depositado sobre o terreno é representada pela Figura 4. 

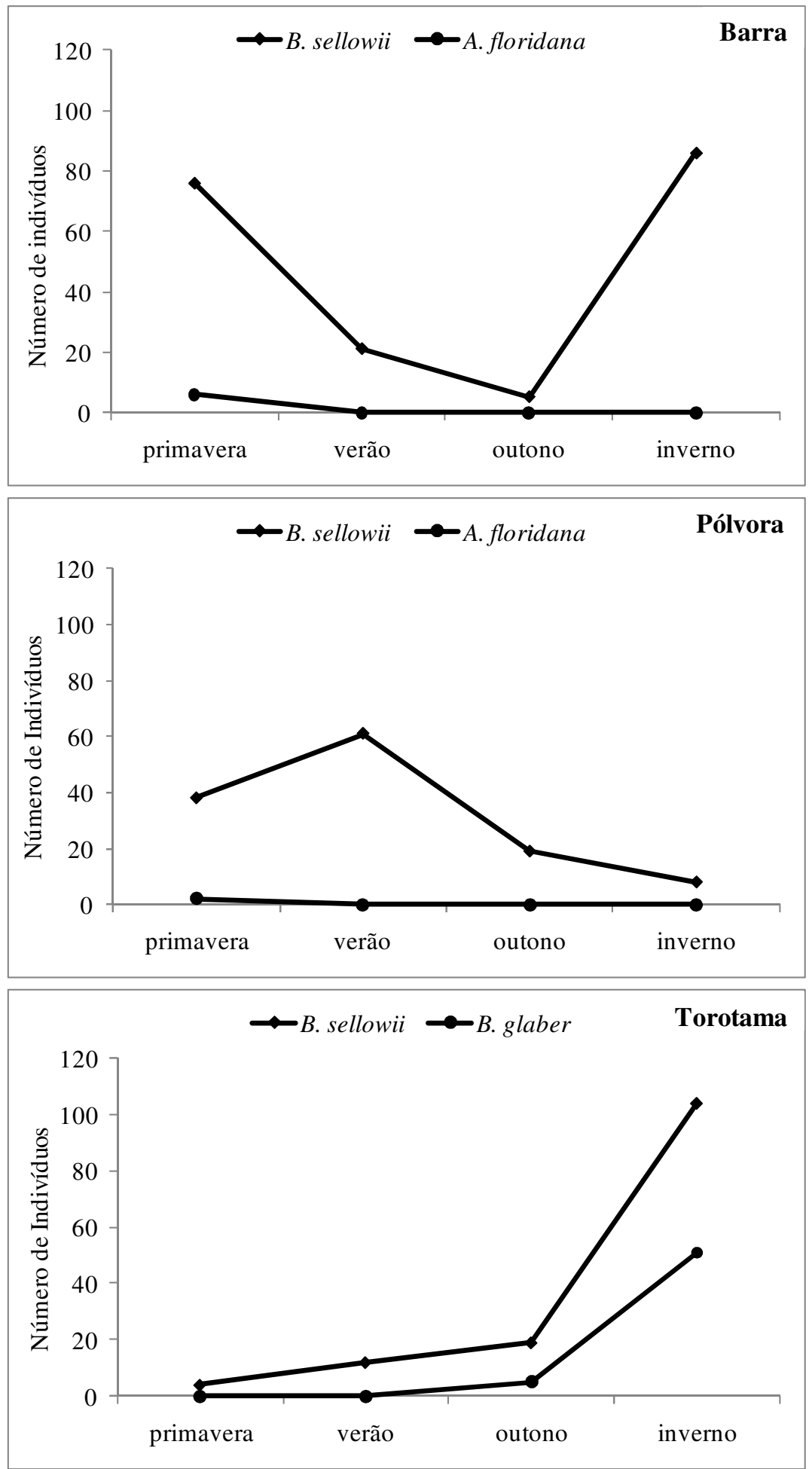

FIGURA 2 - Variação sazonal do número de indivíduos das espécies de isópodos terrestres coletados em três marismas do Estuário da Lagoa dos Patos, RS, no período amostral. 


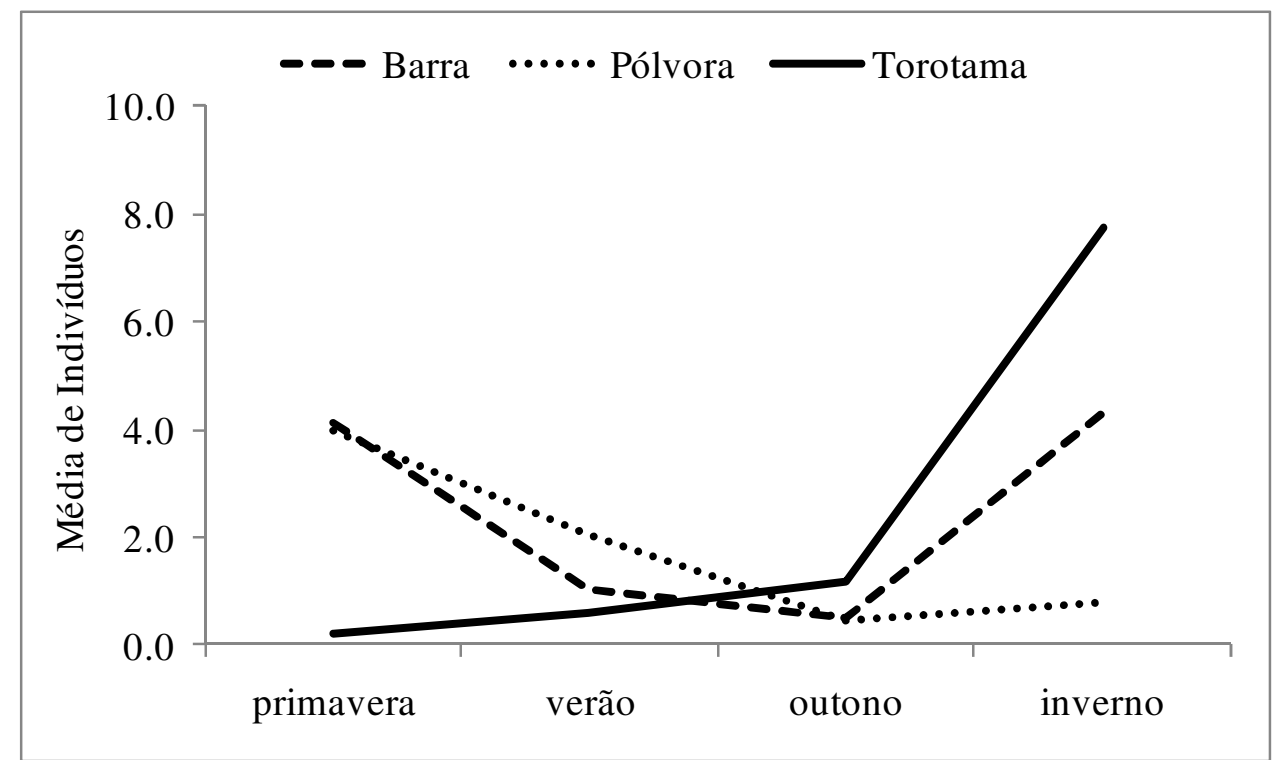

FIGURA 3 - Abundância média de Oniscidea nas marismas do Estuário da Lagoa dos Patos, RS, durante o período amostral.

TABELA I - Espécies de isópodos terrestres coletadas em três marismas do Estuário da Lagoa dos Patos, Rio Grande do Sul, Brasil, durante o período amostral. Total de quadrados amostrados $=470$.

\begin{tabular}{lcccccccc}
\hline & Barra & Hábitat & Pólvora & Hábitat & Torotama & Hábitat & Total & $\%$ \\
\hline B.sellowii & 188 & alta & 119 & alta & 139 & média & 446 & 86,27 \\
& & & 07 & média & & & 07 & 1,35 \\
A.floridana & 06 & alta & 02 & alta & ---- & ---- & 08 & 10,83 \\
B.glaber & ----- & ----- & ---- & ---- & 56 & média & 56 & 1,55 \\
\hline
\end{tabular}



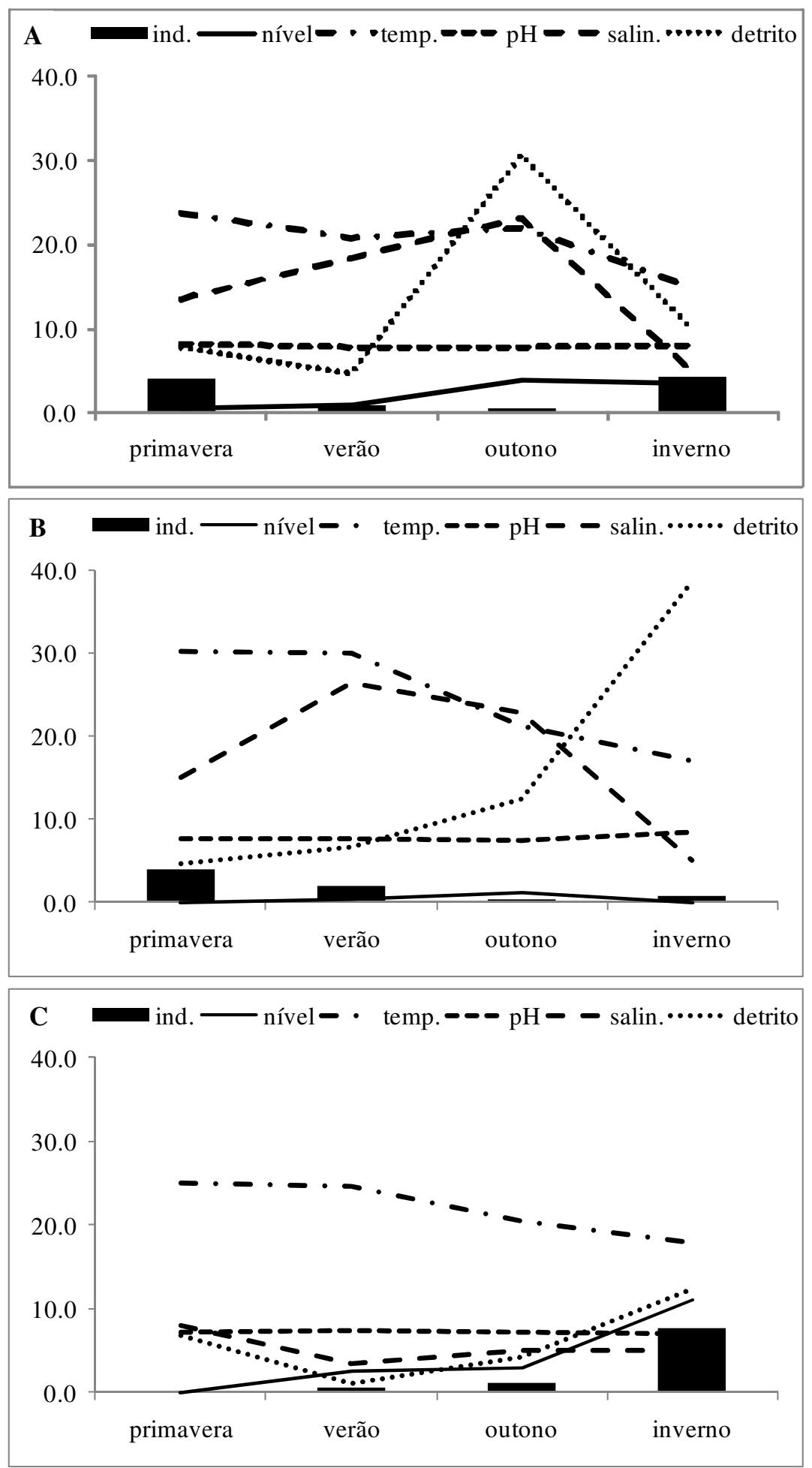

FIGURA 4 - Abundância média de Oniscidea e parâmetros ambientais médios amostrados nas marismas do Estuário da Lagoa dos Patos, durante o período de estudo: A. Barra, B. Pólvora e C. Torotama.

Através da MANOVA, observou-se que a abundância de isópodos terrestres é estatisticamente semelhante nas três marismas amostradas $(p=0,92)$, mas diferente entre as estações do ano $(p=0,00)$. No inverno, o número de indivíduos coletados é significativamente maior do que nas demais estações (Tab. II). A análise também indicou interação entre as variáveis $(p=0,00)$. Após o Teste de Tukey, foi constatado que na Torotama/Inverno, a abundância de isópodos é estatisticamente maior do que nas demais estações e locais, exceto na Barra/Primavera $(p=0,14)$; Barra/Inverno $(p=0,20)$ e Pólvora/Primavera $(p=0,38)$ (Tab. III). 


\begin{tabular}{|c|c|c|c|c|}
\hline & primavera & verão & outono & inverno \\
\hline & 2,52 & 1,57 & 0,96 & 4,98 \\
\hline Primavera & & 0,5922 & 0,2013 & 0,0105 \\
\hline Verão & 0,5922 & & 0,8553 & 0,0000 \\
\hline Outono & 0,2013 & 0,8553 & & 0,0000 \\
\hline Inverno & 0,0105 & 0,0000 & 0,0000 & \\
\hline
\end{tabular}

\begin{tabular}{|c|c|c|c|c|c|c|c|c|c|c|c|c|c|}
\hline \multicolumn{14}{|c|}{$\begin{array}{l}\text { TABELA III - Resultados do Teste de Tukey comparando a abundância de isópodos terrestres coletados } \\
\text { sazonalmente em três marismas do Estuário da Lagoa dos Patos, Rio Grande, Rio Grande do Sul, Brasil } \\
\text { (p<0,05). Marisma: } 1 \text { a } 4 \text { - Barra; } 5 \text { a } 8 \text { - Pólvora; } 9 \text { a } 12 \text { - Torotama. Estação: P - primavera; V - verão; O - } \\
\text { outono; I - inverno. Valores em negrito mostram diferenças estatísticas. }\end{array}$} \\
\hline \multirow[t]{2}{*}{ Marisma } & \multirow[t]{2}{*}{ Estação } & $\{1\}$ & $\{2\}$ & $\{3\}$ & $\{4\}$ & $\{5\}$ & $\{6\}$ & $\{7\}$ & $\{8\}$ & $\{9\}$ & $\{10\}$ & $\{11\}$ & $\{12\}$ \\
\hline & & 4,1 & 1,0 & 0,5 & 4,3 & 4,0 & 2,9 & 0,7 & 0,8 & 0,2 & 0,6 & 1,2 & 7,7 \\
\hline$\{1\}$ & $P$ & & 0,39 & 0,45 & 1,00 & 1,00 & 1,00 & 0,24 & 0,59 & 0,08 & 0,19 & 0,47 & 0,14 \\
\hline$\{2\}$ & V & 0,39 & & 1,00 & 0,29 & 0,75 & 0,95 & 1,00 & 1,00 & 1,00 & 1,00 & 1,00 & 0,00 \\
\hline$\{3\}$ & O & 0,45 & 1,00 & & 0,36 & 0,71 & 0,92 & 1,00 & 1,00 & 1,00 & 1,00 & 1,00 & 0,00 \\
\hline$\{4\}$ & I & 1,00 & 0,29 & 0,36 & & 1,00 & 0,99 & 0,17 & 0,50 & 0,05 & 0,13 & 0,36 & 0,20 \\
\hline$\{5\}$ & $P$ & 1,00 & 0,75 & 0,71 & 1,00 & & 1,00 & 0,61 & 0,82 & 0,36 & 0,54 & 0,81 & 0,38 \\
\hline$\{6\}$ & V & 1,00 & 0,95 & 0,92 & 0,99 & 1,00 & & 0,86 & 0,97 & 0,59 & 0,80 & 0,97 & 0,01 \\
\hline$\{7\}$ & O & 0,24 & 1,00 & 1,00 & 0,17 & 0,61 & 0,86 & & 1,00 & 1,00 & 1,00 & 1,00 & 0,00 \\
\hline$\{8\}$ & I & 0,59 & 1,00 & 1,00 & 0,50 & 0,82 & 0,97 & 1,00 & & 1,00 & 1,00 & 1,00 & 0,00 \\
\hline$\{9\}$ & $P$ & 0,08 & 1,00 & 1,00 & 0,05 & 0,36 & 0,59 & 1,00 & 1,00 & & 1,00 & 1,00 & 0,00 \\
\hline$\{10\}$ & V & 0,19 & 1,00 & 1,00 & 0,13 & 0,54 & 0,80 & 1,00 & 1,00 & 1,00 & & 1,00 & 0,00 \\
\hline$\{11\}$ & $\mathrm{O}$ & 0,47 & 1,00 & 1,00 & 0,36 & 0,81 & 0,97 & 1,00 & 1,00 & 1,00 & 1,00 & & 0,00 \\
\hline$\{12\}$ & 1 & 0,14 & 0,00 & 0,00 & 0,20 & 0,38 & 0,01 & 0,00 & 0,00 & 0,00 & 0,00 & 0,00 & \\
\hline
\end{tabular}

A regressão múltipla mostrou que, para o Molhe Oeste, a salinidade é inversamente proporcional ao número de indivíduos coletados (Tab. IV). Na Ilha da Pólvora, a abundância de isópodos terrestres tende a aumentar com o aumento da temperatura (Tab. V). Outros parâmetros relevantes, mas não estatisticamente significativos, são o aumento da biomassa aérea $(p=0,12)$ e a diminuição da salinidade $(p=0,30)$. Já na llha da Torotama, onde a salinidade e a temperatura são pouco variáveis, o número de isópodos terrestres coletados cresce com o aumento do nível da água e do detrito depositado sobre o terreno (Tab. VI). A biomassa aérea também parece ser relevante, embora não mostre significância estatística $(p=0,18)$. 


\begin{tabular}{|c|c|c|c|c|c|c|c|}
\hline & Beta & Erro padrão & $B$ & Erro padrão & $\mathrm{t}$ & $p$ & $R^{2}$ ajust \\
\hline Interação & & & 5,77 & 1,25 & 4,61 & 0,00 & 0,85 \\
\hline Salinidade & $-0,31$ & 0,12 & $-0,22$ & 0,08 & $-2,73$ & 0,01 & \\
\hline
\end{tabular}

TABELA V - Sumário da análise da regressão múltipla estimada entre o número de indivíduos e parâmetros ambientais registrados para llha da Pólvora, Estuário da Lagoa dos Patos, RS, Brasil.

\begin{tabular}{cccccccc}
\hline & Beta & Erro padrão & $\mathrm{B}$ & Erro padrão & $\mathrm{t}$ & $\mathrm{p}$ & $\mathrm{R}^{2}$ ajust \\
Interação & & & $-1,69$ & 2,58 & $-0,66$ & 0,51 & 0,16 \\
Temperatura & $\mathbf{0 , 3 8}$ & $\mathbf{0 , 1 2}$ & $\mathbf{0 , 2 2}$ & $\mathbf{0 , 0 7}$ & $\mathbf{3 , 1 2}$ & $\mathbf{0 , 0 0}$ & \\
Biomassa & 0,19 & 0,12 & 0,09 & 0,05 & 1,57 & 0,12 & \\
Salinidade & $-0,13$ & 0,12 & $-0,09$ & 0,09 & $-1,05$ & 0,30 & \\
\hline
\end{tabular}

TABELA VI - Sumário da análise da regressão múltipla estimada entre o número de indivíduos e parâmetros ambientais registrados para a llha da Torotama, Rio Grande, Estuário da Lagoa dos Patos, RS, Brasil.

\begin{tabular}{cccccccc}
\hline & Beta & Erro padrão & $\mathrm{B}$ & Erro padrão & $\mathrm{t}$ & $\mathrm{p}$ & $\mathrm{R}^{2}$ ajust \\
Interação & & & $-\mathbf{1 , 5 0}$ & $\mathbf{0 , 5 6}$ & $\mathbf{- 2 , 6 8}$ & $\mathbf{0 , 0 1}$ & $\mathbf{0 , 5 3}$ \\
Nível água & $\mathbf{0 , 4 3}$ & $\mathbf{0 , 0 9}$ & $\mathbf{0 , 4 5}$ & $\mathbf{0 , 0 9}$ & $\mathbf{4 , 9 6}$ & $\mathbf{0 , 0 0}$ & \\
Detrito & $\mathbf{0 , 3 4}$ & $\mathbf{0 , 1 0}$ & $\mathbf{0 , 2 3}$ & $\mathbf{0 , 0 7}$ & $\mathbf{3 , 3 2}$ & $\mathbf{0 , 0 0}$ & \\
Biomassa & 0,14 & 0,10 & 0,10 & 0,08 & 1,36 & 0,18 & \\
\hline
\end{tabular}

\section{DISCUSSÃO}

Balloniscus sellowii foi a única espécie encontrada nas três marismas amostradas, apresentando maiores densidades de indivíduos. Nativa da América do Sul, a espécie se distribui entre os paralelos $20^{\circ}$ e $40^{\circ}$ Sul, ocorrendo preferencialmente nas proximidades do litoral, apresentando uma grande tolerância à variação de salinidade, já tendo sido encontrada em detritos junto à linha de maré e em salinas (LEMOS DE CASTRO 1976). No Rio Grande do Sul é uma espécie considerada muito comum (ARAUJO 1999) e amplamente distribuída pelo Estado (LOPES et al. 2005).

A segunda espécie mais abundante foi Balloniscus glaber, sendo encontrada apenas na marisma da llha da Torotama. B. glaber é uma espécie nativa do Rio Grande do Sul, sendo sua localidade tipo a cidade de Rio Grande (RS), com clima subtropical marítimo e vegetação típica de restinga (ARAUJO \& ZARDO 1995). Segundo LOPES et al. (2005), esta espécie teve sua ocorrência ampliada, sendo registrada desde a Planície Costeira até o Planalto RioGrandense, facilmente encontrada em locais úmidos e com pouca luminosidade. Vive muitas vezes enterrado no solo ou entre as raízes de gramíneas, embaixo de folhiço, pedras e troncos podres (ARAUJO 1999). B. glaber também pode habitar ambientes secos e quentes, embora seja sempre encontrada nos locais mais úmidos destes ambientes (ARAUJO \& ZARDO 1995). Segundo QUADROS \& ARAUJO (2008), esta espécie é abundante próximo à praia, sendo encontrada junto ao detrito ou enterrada na camada superior do solo, no Parque Estadual de Itapuã (RS). Para SCHMALFUSS (1984), está é uma estratégia para suportar as condições climáticas desfavoráveis.

O registro destas espécies já havia sido citado para as marismas do Estuário da Lagoa dos Patos. GAONA et al. (1996) infere as altas taxas de perda de biomassa de detrito da marisma ao processo de utilização deste pelos isópodos terrestres $B$. sellowii e B. glaber, como fonte de alimentação. Para COSTA et al. (1997), os pisos mais elevados das marismas têm como residentes permanentes uma grande diversidade de invertebrados terrestres, entre eles representantes do gênero Balloniscus.

Poucos exemplares de Atlantoscia floridana 
foram encontrados nas marismas do Estuário da Lagoa dos Patos. É uma espécie nativa das Américas, sendo descrita pela primeira vez a partir de espécimes coletados na Flórida (EUA). De acordo com LEMOS DE CASTRO (1958) é o representante de Philosciidae predominante em diferentes habitats, como bromélias, fungos, ninhos de formigas, húmus, paus podres, sob folhas caídas no chão, sob pedras e detritos em geral. É amplamente distribuída pelo Rio Grande do Sul, especialmente na depressão central e na costa marítima (ARAUJO et al. 1996; LOPES et al. 2005). Segundo LEMOS DE CASTRO (1985), A. floridana vive preferencialmente na faixa litorânea, onde o grau de umidade é maior. No entanto, este é o primeiro registro desta espécie para ambientes de marismas.

A densidade média anual de Oniscidea estimada para as marismas do Estuário da Lagoa dos Patos é similar entre os locais amostrados. A densidade populacional de Balloniscus sellowii estimada neste estudo pode ser considerada alta (Barra $=6,72 \mathrm{ind} / \mathrm{m}^{2} ;$ Pólvora $=4,95 \mathrm{ind} / \mathrm{m}^{2} \mathrm{e}$ Torotama $=4,35 \mathrm{ind} / \mathrm{m}^{2}$ ). As densidades de Balloniscus glaber (Torotama $=1,75 \mathrm{ind} / \mathrm{m}^{2}$ ) e de Atlantoscia floridana (Barra $=0,22 \mathrm{ind} / \mathrm{m}^{2}$ e Pólvora $=$ $0,07 \mathrm{ind} / \mathrm{m}^{2}$ ) também estão dentro dos padrões estimados para ambientes de marisma.

DIAS et al. (2005), estimou a densidade de quatro espécies numa marisma do Rio Formosa, Portugal, encontrando forte dominância de Tylos ponticus $\left(29,5 \mathrm{ind} / \mathrm{m}^{2}\right)$ em relação as demais espécies (Porcellio lamellatus $=0,36 \mathrm{ind} / \mathrm{m}^{2} ;$ Armadillidium album $=0,19 \mathrm{ind} / \mathrm{m}^{2}$ e Halophisloscia couchii $=0,03$ ind $/ \mathrm{m}^{2}$ ). HORNUNG \& WARBURG (1995), em florestas e campos na costa mediterrânea, estimaram a densidade populacional de sete espécies de Oniscidea, mas seus valores foram menores que $0,01 \mathrm{ind} / \mathrm{m}^{2}$. No entanto, ZIMMER et al. (2002), registraram altas densidades de isópodos terrestres numa floresta costeira dos Estados Unidos (Venezillo parvus $=60$ ind $/ \mathrm{m}^{2}$; Porcellionides virgatus $=30 \mathrm{ind} / \mathrm{m}^{2} \mathrm{e}$ Littorophiloscia vittata $\left.=<20 \mathrm{ind} / \mathrm{m}^{2}\right)$.

Para DIAS et al. (2005), as condições de estresse do ambiente de marisma impõe uma distinta pressão de seleção para os organismos que lá vivem. Um resultado desta pressão é que enquanto a biodiversidade das marismas é freqüentemente mais baixa quando comparada à ecossistemas marinhos e terrestres adjacentes (KREEGER \& NEWELL 2000; LEVIN \& TALLEY 2000), a abundância das espécies que podem adaptar-se a estas condições tende a ser mais alta (TEAL 1962; MONTAGUE et al. 1981).

A diferença na variação sazonal dos isópodos terrestres nas marismas do Estuário da Lagos dos Patos deve-se às características ambientais de cada habitats. Nas duas marismas mais próximas à água estuarina (Barra e Pólvora), onde a oscilação de maré e salinidade é mais acentuada, a abundância de Oniscidea foi maior na primavera e no inverno, com queda no verão e no outono. Em ambas as marismas, o número de indivíduos tende a ser maior com a diminuição da salinidade. Nos meses de inverno, foi constatada uma grande quantidade de chuva na região, o que pode ter influenciado a diminuição da salinidade e o aumento no número de indivíduos. DESENDER \& MAELFAIT (1999), ao analisarem a diversidade de artrópodos terrestres em um gradiente de salinidade ao longo do Rio Schelde (Bélgica), afirmam que a salinidade é inversamente proporcional à riqueza e à diversidade de espécies. Por outro lado, o período de seca, principalmente durante o verão, é conhecido por causar grande mortalidade em isópodos terrestres (KHEIRALLAH 1979; PARIS 1963; SUTTON 1968).

A abundância dos isópodos terrestres, na llha da Pólvora, diminuiu drasticamente no inverno, pois, devido à quantidade de chuva na região e a proximidade do ponto amostral com a Lagoa, a marisma manteve-se inundada neste período, o que pode ter provocado a morte dos animais por afogamento. Segundo HASSAL \& DANGERFIELD (1997), a sobrevivência e a densidade populacional de Armadillidium vulgare Latreille, 1804, são negativamente correlacionadas com a precipitação, devido à umidade excessiva do solo. Para os autores, quando a água do solo não é congelada pelo frio intenso, pode ocorrer aumento da mortalidade de isópodos terrestres, por afogamento.

A llha da Torotama é considerada como uma das marismas mais importantes para o Estuário da Lagoa dos Patos, devido a sua grande extensão (COSTA et al. 1997). Como é uma enseada mais protegida e localizada no interior do estuário, a salinidade é pouco variável, permanecendo entre 5 e 10. Com isto, este parâmetro não apresenta influência 
sobre a abundância dos isópodos. Os parâmetros que contribuíram para a elevação da abundância de isópodos terrestres na llha da Torotama foram a elevação do nível da água e o detrito depositado sobre o terreno, além da maior quantidade de biomassa viva. Para ZIEMAN \& ZIEMAN (1989), o valor de uma unidade pode ser determinado pela abundância e variabilidade espacial da biomassa vegetal (viva e morta), que governa a quantidade de recurso alimentar disponível, o grau de proteção contra predadores e a área de substrato disponível. Segundo DIAS et al. (2005), as espécies de isópodos terrestres analisadas nas marismas do Rio Formosa (Portugal), são mais abundantes em marismas ocasionalmente alagadas, como forma de evitar as condições de dessecação. Outro fato levantado pelos autores, é que, na marisma média, há maior quantidade de detrito acumulado, no ou sob o qual as espécies se escondem.

Tais diferenças na abundância dos isópodos terrestres nas marismas do Estuário da Lagoa dos Patos são corroboradas pelas informações de NETO \& LANA (1994), que afirmam que áreas entremarés tropicais ou subtropicais podem apresentar alta abundância de invertebrados no verão e no inverno. Já em regiões temperadas, a maior abundância de organismos invertebrados ocorre no final da primavera e início do verão (RUTLEDGE \& FLEEGER 1993; BALLA \& DAVIS 1995). Segundo SZLÀVECZ (1995), a diversidade e abundância de Oniscidea esta positivamente relacionada com as condições de umidade dos ambientes. Para FERRARA \& TAITI (1998), a distribuição e abundância de isópodos terrestres são fortemente influenciadas por fatores ambientais, onde a maioria das espécies encontra-se em áreas menos extremas. DESENDER \& MAELFAIT (1999), afirmam que a maior diversidade de artrópodos terrestres é registrada em ambientes afastados do mar.

\section{AGRADECIMENTOS}

Os autores agradecem os estagiários Kelen Veiga, Marcelo Nunes e Thais Soler pelo auxílio nas coletas e triagem do material e ao CNPq, pela bolsa de Pós-doutorado do primeiro autor.

\section{LITERATURA CITADA}

ADAM, P. 1993. Salthmarsh ecology. New York, Cambridge University Press, 461p.

ARAUJO, PB. 1999. Two new species of Alboscia Schultz, 1995 from Rio Grande do Sul, Brasil (Isopoda, Oniscidea, Philosciidae). Crustaceana, 72(5): 487-496.

ARAUJO, PB. \& CML ZARDO. 1995. Uma nova espécie de Balloniscus Budde-Lund (Crustacea, Isopoda, Balloniscidae) do sul do Brasil. Revta. bras. Zool., 12(4): 785-790.

ARAUJO, PB., L BUCKUP \& G BOND-BUCKUP. 1996. Isópodos terrestres de Santa Catarina e Rio Grande do Sul (Crustacea, Oniscidea). Iheringia, 81: 111-118.

BALLA, SA. \& JA DAVIS. 1995. Seasonal variation in macroinvertebrate fauna of wetlands of differing water regime and nutrient status on the Swan coastal plain, Western Australia. Hydrobiologia, 299: 147-161.

CHAPMAN, V.J. 1992. Ecosystems of the World - west coastal ecosystems. Amsterdam, Elsevier Science Publishers, 428p.

COSTA, C.S.B. 1998. Marismas Irregularmente Alagadas. In Os Ecossistemas Costeiro e Marinho do Extremo Sul do Brasil (SEELIGER, U, C ODEBRECHT \& JP CASTELLO, eds), Rio Grande: Ecoscientia. pp. 82-85.

COSTA, CSB. \& AJ DAVY. 1992. Development and organization of salt marsh communities. In Coastal Plant Communities of Latin America (SEELIGER, U, ed). New York: Academic Press. pp. 157-178.

COSTA, CSB., U SEELIGER, CPL OLIVEIRA \& AMM MAZO. 1997. Distribuição, funções e valores das marismas e pradarias submersas no estuário da Lagoa dos Patos (RS, Brasil). Atlântica, 19: 65-83.

COSTA, CSB., JC MARANGONI \& AMG AZEVEDO. 2003. Plant zonation in irregularly flooded sat marshes: relative importance of stress tolerance and biological interaction. J. Ecol., 91(6): 951-965.

COIMBRA, F. \& CSB COSTA. 2006. Mapeamento digital dos macrohábitats de dunas e marismas da costa do Rio Grande do Sul através de imagens de satélite e fotografias aéreas. In (ENCOGERCO, ed), Santos: Agência Brasileira de Gerenciamento Costeiro, pp. 1-4.

DIAS, N., M SPRUNG \& M HASSALL. 2005. The abundance and life histories of terrestrial isopods in a salt marsh of the Ria Formosa lagoon system, southern Portugal. Mar. Biol., 147: 1343-1352.

DESENDER, K. \& J-P MAELFAIT. 1999. Diversity and conservation of terrestrial arthropods in tidal marshes along the River Schelde: a gradient analysis. Biol. Conserv., 87: 221-229.

FERRARA, F. \& S TAITI. 1998. Biogeography of the Oniscidean fauna of Somalia. Isr. J. Zool., 44: 283-290.

GAONA, CAP., AR PEIXOTO \& CSB. COSTA. 1996. Produção primária de uma marisma raramente alagada dominada por Juncus effusus L., no extremo sul do Brasil. Atlântica, 18: 43-54.

HASSALL, M. \& JM DANGERFIELD. 1997. The population dynamics of a woodlouse, Armadillium vulgare: an example of biotic compensatory mechanisms amongst terrestrial macrodecomposers? Pedobiologia, 41: 342-360.

HICKENBICK, GR., AL FERRO \& PC ABREU. 2004. Produção de detrito de macrófitas emergentes em uma marisma do Estuário da Lagoa dos Patos: taxas de decomposição e dinâmica 
microbiana. Atlântica, 26(1): 61-75.

HORNUNG, E. \& MR WARBURG. 1995. Seasonal changes in the distribution and abundance of isopod species in different habitats within the Mediterranean region of Northern Israel. Acta Oecol., 16(4): 431-445.

KHEIRALLAH, AM. 1979. The ecology of the isopods Perisoyphis granai (Arcangeli) on the western highlands of Saudi Arabia. J.Arid Environ., 2: 51-59.

KREEGER, DA. \& RIE NEWELL. 2000. Trophic complexity between producers and invertebrate consumers in salt marshes. In Concepts and controversies in tidal marsh ecology (WEINSTEIN, MP \& DA KREEGER, eds), Dordrecht: Kluwer. pp. 187-220.

LEMOS DE CASTRO, A. 1958. On the systematic position of some american species of Philoscia Latreille (Isopoda, Oniscoidea). Amer. Mus. Nov., 1908: 1-10.

LEMOS DE CASTRO, A. 1976. Considerações sobre a sinonímia e a distribuição geográfica de Balloniscus sellowii (Brandt, 1833) (Isopoda, Balloniscidae). Revta bras. Biol., 36(2): 391-396.

LEMOS DE CASTRO, A. 1985. Considerações sobre Atlantoscia alceui Ferrara \& Taiti, 1981 (Isopoda, Oniscoidea, Philosciidae). Revta bras. Biol., 45(4): 417-442

LOPES, ERC., MS Jr MENDONÇA, G BOND-BUCKUP \& PB ARAUJO. 2005. Oniscidea diversity across three environments in an altitudinal gradient in northeastern Rio Grande do Sul, Brasil. Eur. J. Soil Biol., 41: 99-107.

LEVIN, LA. \& TS TALLEY. 2000. Influences of vegetation and abiotic environmental factors on salt marsh invertebrates. In Concepts and controversies in tidal marsh ecology (WEINSTEIN, MP \& DA KREEGER, eds), Dordrecht: Kluwer. pp. 661-707

MONTAGUE, C.L.; BUNKER, S.M.; HAINES, E.B.; PACE, M.L. and WETZEL, R.L. 1981. Aquatic macroconsumers. p. 69-86. In: L.R. Pomeroy and Wiegert, R.G. (eds), The ecology of a salt marsh. New York, Springer, Berlin Heidelberg.

NETO, SA. \& PD LANA. 1994. Effects of sediment distubance on the structure of benthic fauna in a subtropical tidal creek of southeastern Brazil. Mar. Ecol. Progr. Ser., 106(3): 239-247.

PANITZ, CMN. 1992. Ecological aspects of a salt marsh ecosystem in Santa Catarina island, Brazil. In Coastal Vegetation in Latin América (SEELIGER, U, ed), New York: Academic Press. pp. 213-230.

PARIS, OH. 1963. The ecology of Armadillidium vulgare (Isopoda: Oniscoidea) in California grassland: food, enemies and weather. Ecol. Monogr., 33: 1-22.

QUADROS, AF. \& PB ARAUJO. 2008. An assemblage of terrestrial isopods (Crustacea) in southern Brazil and its contribution to leaf litter processing. Revta bras. Zool., 25(1): 58-66.

RUTLEDGE, PA. \& JW FLEEGER. 1993. Abundance e seasonality of meiofauna, including harpacticoid copepod species, associated with stems of the salt-marsh cord grass, Spartina alterniflora. Estuaries, 16:760-768.

SCHMALFUSS, H. 1984. Eco-morphological strategies in terrestrial isopods. Symposia of the Zoological Society of London, 53: 339-368.

SEELIGER, U., CSB COSTA \& PC ABREU. 1997. Primary production cycles. In Subtropical Convergence Environments: the Coast and Sea in the Southwestern Atlantic (SEELIGER, U, C ODEBRECHT \& JP CASTELLO, eds), Berlin: SpringerVerlag. pp. 65-70.

SILVA, CP., CMP PEREIRA \& LPD DORNELES. 1993. Espécies de gramíneas e crescimento de Spartina densiflora Brong. em uma marisma da Laguna dos Patos, RS, Brasil. Caderno de Pesquisa Série Botânica, 5(1): 95-108.

SUTTON, SL. 1968. The population dynamics of Trichoniscus pusillus and Philoscia muscorum (Crustacea, Oniscoidea) in limestone grassland. J. Anim. Ecol., 37: 425-444.

SZLAVECZ, K. 1995. Diversity and spatial community structure of terrestrial isopods (Isopoda, Oniscidea) in a mosaic of plant assemblage. In Terrestrial Isopod Biology (ALIKAN, MA, ed). In Crustacean Issues (SCHRAM, FR, ed), Rotterdam. pp. 97-107.

TALLEY, TS., JA CROOKS \& LA LEVIN. 2001. Habitat utilization and alteration by the invasive burrowing isopod, Sphaeroma quoyanum, in California salt marshes. Mar. Biol., 138: 561-573.

TEAL, JM. 1962. Energy flow in the salt marsh ecosystem of Georgia. Ecology, 43(4): 614-624.

VERNBERG, FJ. 1993. Salt-marsh processes: a review. Envir. Toxicol. Chem., 12: 2167-2193.

ZAR, JH. 1999. Biostatistical Analysis. New Jersey, Prentice-Hall, $931 \mathrm{p}$.

ZIEMAN, JC. \& RT ZIEMAN. 1989. The ecology of the seagrass meadows of the west coast of Florida: a community profile. Biol. Repres., 85: 7-25.

ZIMMER, M. 2003. Habitat and resource use by terrestrial isopods (Isopoda: Oniscidea). In The biology of terrestrial isopods. Proceedings of the $5^{\text {th }}$ international symposium on the biology of terrestrial isopods. (SFENTHOURAKIS, S., PB ARAUJO, E HORNUNG, H SCHMALFUSS, S TAITI \& S SZLAVECZ, eds), Crustaceana Monographs. pp. 243-261.

ZIMMER, M., SC PENNINGS, TL BUCK \& TH CAREFOOT. 2002. Species-specific patterns of litter processing by terrestrial isopods (Isopoda: Oniscidea) in high intertidal salt marshes and coastal forests. Func. Ecol., 16: 596-607. 